\title{
Molecular orbital studies on the Wagner-Meerwein migration in some acyclic pinacol-pinacolone rearrangements
}

\author{
ZODINPUIA PACHUAU and R H DUNCAN LYNGDOH* \\ Department of Chemistry, North-Eastern Hill University, Shillong 793 022, India \\ e-mail: rhdl@nehu.ac.in
}

MS received 15 March 2003; revised 15 October 2003

\begin{abstract}
The semi-empirical PM3 SCF-MO method is used to investigate the Wagner-Meerwein migration of various groups during the pinacol-pinacolone rearrangement of some acyclic systems. Pinacol first protonates and dehydrates to form a carbocation that undergoes a 1,2-migration to form a protonated ketone, which then deprotonates to yield the pinacolone product. We study the Wagner-Meerwein migration of hydride, methyl, ethyl, isopropyl, $t$-butyl, phenyl and heterocylic 2-, 3- and 4-pyridyl groups in various acyclic 1,2-diol (pinacol) systems as they rearrange to pinacolones. This 1,2-migration involves a three-centred moiety in the cationic transition state. The migratory aptitude predicted here follows the order: hydride $>t$-butyl $>$ isopropyl $>$ ethyl $>$ methyl $>$ phenyl, which accords well with available experimental data and/or chemical intuition, reflecting also on the ability of the group involved to carry positive charge in the transition state. The structure of the migrating group (whether aliphatic or aromatic) within the transition state also supports the stabilising role of delocalisation of positive charge for reaction feasibility. Geometrical and thermodynamic considerations coincide in assigning the following order to relative "earliness" of the transition state along the reaction pathway: $t$-butyl $>$ isopropyl > phenyl > methyl > 2-pyridyl > 4-pyridyl.
\end{abstract}

Keywords. Pinacol-pinacolone rearrangement; Wagner-Meerwein migration; relative migratory aptitude; transition state geometry; Hammond's postulate; PM3 SCF-MO method.

\section{Introduction}

Carbocation existence, although often only transient, has been the subject of intense experimental and theoretical study in relation to structure, stability and reactivity (including rearrangements), ${ }^{1}$ having acquired a prominent and interesting place in the world of chemistry. Their widespread occurrence as reactive intermediates in chemical reactions enables carbocations to be of considerable importance in a large variety of well-known chemical reactions. ${ }^{2} \mathrm{We}$ concern ourselves here with theoretical studies on structural and reactivity aspects of the WagnerMeerwein migration reaction as applied to the pinacol-pinacolone rearrangement in acyclic systems, which is important in the context of carbocationic species acting as reaction intermediates.

*For correspondence

\subsection{Wagner-Meerwein migrations in pinacol- pinacolone rearrangements}

The Wagner-Meerwein migration, originally studied with respect to secondary and tertiary alcohols derived from terpene systems, now has a much wider scope embracing any 1,2-nucleophilic shift of hydride, alkyl, aryl, aralkyl groups etc within a carbocation or electrophile leading to rearranged products. Pine ${ }^{3}$ has defined it as any migration to an electron-deficient carbon atom, while Sykes ${ }^{4}$ defined it as any change in a carbon skeleton involving carbocations. The Wagner-Meerwein rearrangement in its broadest scope thus incorporates a wide variety of substrates and electrophilic reagents or intermediates. In a typical case of this reaction, an alcohol substrate protonates and eliminates water to create a carbocation species. This carbocation then undergoes the 1,2-nucleophilic group migration leading to a rearranged carbocation, which step constitutes the actual Wagner-Meerwein migration. The new carbocation may then go on to react in various ways, e.g. loss of a carbon-bonded proton (as per Zaitsev's 
rule) to give olefin, or maybe deprotonation from a hetero-atom (e.g. oxygen, if present) to give multiple-bonded products like ketones or imines.

Known since $1860,{ }^{5}$ the pinacol-pinacolone rearrangement has been subjected to much experimental research ${ }^{6}$ pertaining to its stereochemistry ${ }^{7-10}$ and the relative migratory ${ }^{11-14}$ aptitudes of various groups. Pinacols themselves are made by the reduction of ketones with bivalent metals, usually magnesium, under anhydrous conditions. This rearrangement is portrayed in figure 1 for the typical case of a 1,2,2trimethyl-1,2,-diol, 1 (known as a pinacol) which bears a substituent $\mathrm{R}$ at the 1-position. Protonation occurs at one of the hydroxy groups in acidic medium to give rise to species 2 . This then eliminates water to give an $\alpha$-hydroxy carbocation 3 . The group $R$ then undergoes 1,2 shift from the $\alpha$-carbon of $\mathbf{3}$ to its carbocation centre via a transition state $\mathbf{4}$, forming a rearranged cation $\mathbf{5}$, which then $\mathrm{O}$-deprotonates yielding a ketone $\mathbf{6}$ as the final rearrangement product (a pinacolone). This rearrangement can proceed with 1,2-diol substrates (pinacols) containing alkyl, aryl, hetaryl or aralkyl groups. Different groups $\mathrm{R}$ have varying migratory aptitudes, estimation of which forms a part of this study.

\subsection{Approach to this study}

To date, no significant theoretical work has been carried out to elucidate mechanistic aspects of the pinacol-pinacolone rearrangement, thereby prompting this study incorporating a set of nine different pinacols as they arrange to their pinacolone products. Each pinacol 1 is a 1-substituted 1,2,2-trimethyl1,2,-diol with the 1-substituent $\mathrm{R}$ taken as the migrating group, where $R$ is respectively hydride $(H)$,

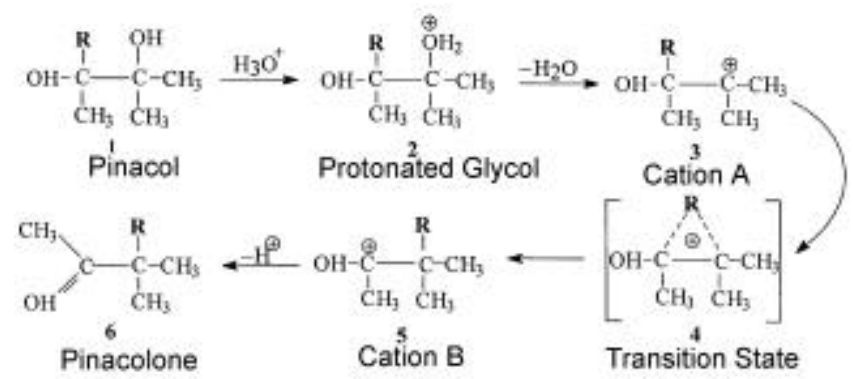

Figure 1. Successive steps of the pinacol-pinacolone rearrangement involving Wagner-Meerwein 1,2 shifts for nine 1,2,2-trimethyl-1,2-diol cases $\left(\mathrm{R}=\mathrm{H}, \mathrm{Me}, \mathrm{Et}, \mathrm{Pr}^{i}\right.$, $\mathrm{Bu}^{t}, 2-\mathrm{Py}, 3-\mathrm{Py}$ and 4-Py). methyl (Me), ethyl (Et), isopropyl ( $\left.\operatorname{Pr}^{i}\right), t$-butyl $\left(\mathrm{Bu}^{t}\right)$, phenyl $(\mathrm{Ph})$, 2-pyridyl (2-Py), 3-pyridyl (3Py) and 4-pyridyl (4-Py) for the nine different cases. Except for the cases where $\mathrm{R}$ is $\mathrm{H}$ or $\mathrm{Me}$, all these pinacols are optically active, while the pinacolone product is optically inactive. For the purpose of this study, we take only the R-isomers to trace the entire course of the rearrangement reaction in each case, leading to the pinacolone product which is itself optically inactive. The corresponding $\mathrm{S}$-isomers in each case would have their reaction courses energetically identical to the R-isomers, since the two reaction courses are simply mirror images of each other, both leading to the same pinacolone product. Apart from the energetics of the entire reaction course, we also investigate in detail the key step of this rearrangement reaction, viz. the 1,2-migration step in the $\alpha$-hydroxy carbocation 3 leading to the rearranged carbocation 5 via the transition state 4 .

1.2a Energy profile of the whole reaction: $\mathrm{By}$ calculating the theoretical heats of formation for each of the species involved in the various steps of the pinacol-pinacolone rearrangement as given in figure 1 (including the transition state $\mathbf{4}$ between $\mathbf{3}$ and 5) we can plot an energy profile diagram for the reaction as a whole for any particular case and make comparisons between the cases studied.

1.2b Relative migratory aptitudes: Limited experimental data ${ }^{15-17}$ on the pinacol-pinacolone rearrangement suggests the order hydride $>$ methyl $>$ phenyl for relative migratory facility. A theoretical study ${ }^{18}$ on bonding between various cations and the ethylene molecule suggested the order $t$-butyl > isopropyl > ethyl > methyl for relative migratory aptitude in a cationic situation. By incorporating different migrating groups $\mathrm{R}$ in the 1-position of the pinacol 1, their migratory aptitudes during the 1,2migration step may be compared. The kinetic facility of migration is estimated by the activation energy for this step, while the thermodynamic facility is estimated simply from the enthalpy of the migration reaction involving conversion of species $\mathbf{3}$ to species 5.

1.2c Transition state geometries: We focus on the transition state 4 involved in the rearrangement of species $\mathbf{3}$ to species $\mathbf{5}$. The transition state geometry may be related to the reactant and product geometries as per the Hammond postulate ${ }^{19}$ which 
states that, for any reaction with a moderate activation barrier and reaction enthalpy, the transition state geometry resembles the reactant geometry for exothermic reactions and the product geometry for endothermic reactions. Exothermic reactions thus have transition states described as "early", while those for endothermic reactions are described as "late". Geometry characteristics of the transition state as computed in this study may help define its position along the reaction coordinate, whether "early", "midway" or "late". These may further be related to the calculated exothermicity or endothermicity of the reaction in question.

\section{Theoretical methodology}

The semi-empirical PM3 SCF-MO method ${ }^{20}$ of the MOPAC 6.0 package $^{21}$ was used to obtain the wavefunction and all molecular properties for all the molecular systems 1 to $\mathbf{6}$ studied here. All equilibrium geometries were obtained by full optimisation without symmetry constraints using the BFGS minimization algorithm. ${ }^{22}$ In each case of the migration step, the reactant $\mathbf{3}$ and product 5 were subjected to conformational analysis to yield the most stable conformer of both. The transition state $\mathbf{4}$ was located for each case by the SADDLE keyword of the MOPAC package, invoking a reverse search strategy with interpolation between the equilibrium geometries of the reactant $\mathbf{3}$ and product $\mathbf{5}$ (in their lowest energy conformers) to arrive at the saddle point or transition state 4. Once located, the transition state was verified as such by diagonalising the Hessian matrix to yield one and only one negative eigenvalue.

\subsection{Enthalpy indices}

The thermodynamics of the various steps along the pathway of the pinacol-pinacolone rearrangement was traced by assigning enthalpy indices to each step as portrayed in figure 1 . The initial step of $\mathrm{O}$ protonation of pinacol $\mathbf{1}$ to give $\mathbf{2}$ is described by the enthalpy of protonation $\Delta H_{p}$ where the pinacol is protonated by a hydronium ion $\mathrm{H}_{3} \mathrm{O}^{+}$. The next step of loss of water from 2 is described by the enthalpy of dehydration $\Delta H_{d h}$ to create the carbocation 3 . This then rearranges to the cation $\mathbf{5}$ via the transition state 4, and the enthalpy of this 1,2-migration step is termed $\Delta H_{r}$. The rearranged species 5 then deprotonates to the pinacolone product $\mathbf{6}$, and this step is described by the deprotonation enthalpy $\Delta H_{d p}$ where the proton lost is accepted by a water molecule. Finally, the overall enthalpy of the pinacol-pinacolone rearrangement is represented by $\Delta H_{p c}$, the enthalpy difference between the reactants (pinacol plus $\mathrm{H}_{3} \mathrm{O}^{+}$) and the final products (pinacolone plus $\mathrm{H}_{3} \mathrm{O}^{+}$and $\mathrm{H}_{2} \mathrm{O}$ ).

\subsection{Relative migratory aptitudes}

Relative migratory aptitude was considered from the kinetic as well as the thermodynamic points of view. The final outcome, however, is expected to depend primarily on the kinetic factor owing to the instability of the cationic reactive intermediates involved. The nine different migrating groups $\mathrm{R}$ were compared with regard to kinetic facility of undergoing the 1,2-migration step by comparing the calculated values of the activation energy $E_{a}$ for the conversion of species $\mathbf{3}$ to species $\mathbf{5}$ for each case. The thermodynamic facility of the migration was also estimated for each case by the enthalpy term $\Delta H_{r}$. The capacity of the migrating group $\mathrm{R}$ to retain charge in the transition state is estimated by calculating the sum total $Q_{r}$ of all the Mulliken atomic charges on the atoms of the group $\mathrm{R}$ as it exists in the transition state.

\subsection{Transition state geometries}

We focus primarily on the three-centered moiety in the transition state $\mathbf{4}$ for the step of 1,2-migration as depicted in figure 2, which consists of the carbon $\mathrm{C} 1$ on the migration origin, the carbon $\mathrm{C} 2$ at the mi-

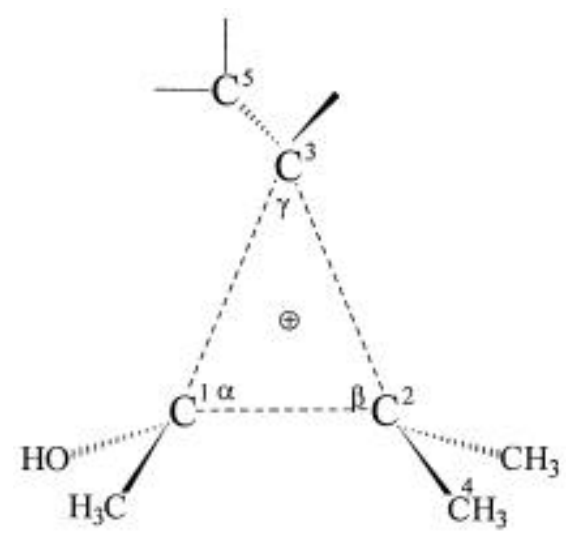

Figure 2. Schematic diagram of the transition state 4 for the Wagner-Meerwein migration step, with dihedral angles $\varphi_{1}=[\mathrm{C} 3-\mathrm{C} 1-\mathrm{C} 2-\mathrm{C} 4]$ and $\varphi_{2}=[\mathrm{C} 1-\mathrm{C} 2-\mathrm{C} 3-\mathrm{C} 5]$. 
gration terminus and the carbon $\mathrm{C} 3$ of the migrating group. This moiety has three $\mathrm{C}-\mathrm{C}$ bond lengths $R_{12}$, $R_{13}$ and $R_{23}$ as shown, of which the C1-C3 bond is being broken while the $\mathrm{C} 3-\mathrm{C} 2$ bond is being formed during the course of the migration. The angles $\alpha, \beta$ and $\gamma$ are defined as the bond angles $\mathrm{C} 2-\mathrm{C} 1-\mathrm{C} 3$, $\mathrm{C} 1-\mathrm{C} 2-\mathrm{C} 3$ and $\mathrm{C} 2-\mathrm{C} 3-\mathrm{C} 1$ respectively. The dihedral angle $\varphi_{1}$ is the one that encompasses the atoms $\mathrm{C} 3, \mathrm{C} 1, \mathrm{C} 2$ and $\mathrm{C} 4$ in turn, where $\mathrm{C} 4$ is the carbon of the 2-methyl group trans to the 1-hydroxy group. Another dihedral angle $\varphi_{2}$ is defined as that encompassing the atoms $\mathrm{C} 1, \mathrm{C} 2, \mathrm{C} 3$ and $\mathrm{C} 5$ in turn, where $\mathrm{C} 5$ is the carbon (or hydrogen) atom bonded to the migrating group carbon $\mathrm{C} 3$ and occurring closest to the plane of the triangular moiety $\mathrm{C} 1-\mathrm{C} 2-\mathrm{C} 3$ and behind it when positioned as in figure 2 and viewed from above. These two dihedrals give some picture of the three-dimensional structure of the transition state.

The transition state geometry may also give clues to the relative position of the transition state along the reaction pathway. We propose the ratio $R_{13} / R_{23}$ of the length of the breaking bond to that of the forming bond as a putative index to help indicate this position along the reaction coordinate. Smaller values of this ratio would indicate the transition state as relatively "early", while larger values would indicate it as relatively "late". These assignments of "early" or "late" may then be compared with the degree of exothermicity or endothermicity of the reaction to validate Hammond's postulate. It must be remembered, though, that this bond length ratio index can only point to relative position along the reaction coordinate in the context of the whole set of cases studied, and does not furnish an index for absolute position along the reaction pathway.

\section{Results and discussion}

This theoretical study has been pursued in the gas phase without consideration of solvent effects, since incorporation of these effects appreciably increases the computational load. The occurrence of the pinacol-pinacolone rearrangement in gas phase is known for unsubstituted pinacol itself. ${ }^{23}$ It is expected that solvation would appreciably increase the stability of the cationic intermediates $\mathbf{3}$ and $\mathbf{5}$ as well as that of the cationic transition state 4 . This would result in an overall decrease in the values of the activation energies for the Wagner-Meerwein migration step, but the order of magnitudes for the activation energies with respect to migrating group may be expected to be the same as for the gas phase, leading to the same conclusions. Likewise, the enthalpy of deprotonation of the intermediate 5 would be expected to decrease upon changing from the gas phase to the solvent phase.

Our study investigates (a) the overall thermodynamic profile of the rearrangement of the acyclic 1,2,2-trimethyl-1,2-diol or pinacol $\mathbf{1}$ to the pinacolone 6, (b) the relative migratory aptitude of the various groups $\mathrm{R}$ as they migrate during rearrangement of species $\mathbf{3}$ to species $\mathbf{5}$, and (c) the geometry of the corresponding transition state $\mathbf{4}$ in the context of the Hammond postulate. This study is carried out for nine different pinacols (1-substituted 1,2,2trimethyl-1,2-diols) with nine different migrating groups $\mathrm{R}$ present as the 1-substituent in the parent pinacol.

\subsection{Overall thermodynamic profile}

Our PM3 enthalpies for four successive steps of the pinacol-pinacolone rearrangement are presented in table 1 for nine different cases $\left(\mathrm{R}=\mathrm{H}, \mathrm{Me}, \mathrm{Et}, \mathrm{Pr}^{i}\right.$, $\mathrm{Bu}^{t}, \mathrm{Ph}, 2-\mathrm{Py}, 3-\mathrm{Py}$ and 4-Py), where the enthalpy indices are as defined earlier, including the overall enthalpy of whole rearrangement. The values for $\Delta H_{p}$, the enthalpy of initial protonation of the pinacol 1 to give 2 , are all negative, ranging from -36.73 to $-22.11 \mathrm{kcal} / \mathrm{mol}$, uniformly indicating the exothermicity of this step. The next step of dehydration of the protonated pinacol $\mathbf{2}$ is predicted to range bet-

Table 1. $P M 3$ enthalpies* for successive steps of the pinacol-pinacolone rearrangement, including enthalpies for the steps of protonation of $\mathbf{1}\left(\Delta H_{p}\right)$, dehydration of $\mathbf{2}$ $\left(\Delta H_{d h}\right)$, rearrangement of $\mathbf{3}\left(\Delta H_{r}\right)$, deprotonation of $\mathbf{5}$ $\left(\Delta H_{d p}\right)$ and for the overall rearrangement of 1 to $6\left(\Delta H_{p c}\right)$ for nine different migrating groups $\left(\mathrm{R}=\mathrm{H}, \mathrm{Me}, \mathrm{Et}, \mathrm{Pr}^{i}\right.$, $\mathrm{Bu}^{t}, \mathrm{Ph}, 2-\mathrm{Py}, 3-\mathrm{Py}$ and 4-Py).

\begin{tabular}{lcrccc}
\hline $\mathrm{R}$ & $\Delta H_{p}$ & $\Delta H_{d h}$ & $\Delta H_{r}$ & $\Delta H_{d p}$ & $\Delta H_{p c}$ \\
\hline $\mathrm{H}$ & -22.11 & 6.24 & -15.64 & 30.69 & -0.82 \\
$\mathrm{Me}$ & -31.50 & 12.93 & -16.22 & 32.14 & -2.65 \\
$\mathrm{Et}$ & -32.24 & 11.50 & -14.92 & 32.93 & -2.73 \\
$\mathrm{Pr}^{i}$ & -32.81 & 7.84 & -13.82 & 34.66 & -4.13 \\
$\mathrm{Bu}^{t}$ & -32.52 & 4.51 & -13.28 & 35.19 & -6.10 \\
$\mathrm{Ph}$ & -31.13 & 6.48 & -15.61 & 34.35 & -5.91 \\
$2-\mathrm{Py}$ & -36.73 & 13.48 & -20.86 & 39.26 & -4.85 \\
$3-\mathrm{Py}$ & -33.04 & 13.12 & -16.72 & 31.61 & -5.03 \\
4-Py & -28.59 & 9.62 & -15.98 & 30.29 & -4.66 \\
\hline
\end{tabular}

*All enthalpies in $\mathrm{kcal} / \mathrm{mol}$ 
ween 4.51 to $13.48 \mathrm{kcal} / \mathrm{mol}$, indicating rather small absolute values for the enthalpy index $\Delta H_{d h}$ here. The cases where $\mathrm{R}$ is an aromatic ring have the enthalpy index $\Delta H_{d h}$ values ranging towards the higher side than the cases where $\mathrm{R}$ is aliphatic. The third step of the rearrangement itself (Wagner-Meerwein migration of group R) is marked by a significant exothermicity, where the enthalpy index $\Delta H_{r}$ ranges from -20.86 to $-13.28 \mathrm{kcal} / \mathrm{mol}$. This indicates a definite thermodynamic favourability for the rearrangement itself, and predicts that the new bond formed between the migrating group $\mathrm{R}$ and the migration terminus $\mathrm{C} 2$ would be significantly stronger than the old one between the group $\mathrm{R}$ and the migration origin $\mathrm{C} 1$.

The enthalpy of deprotonation $\Delta H_{d p}$ to yield the pinacolone product $\mathbf{6}$ is invariably on the positive side, which is parallel to the initial step of protonation of the pinacol 1 where the corresponding protonation enthalpy $\Delta H_{p}$ is always negative. When we come to the overall reaction, viz. the transformation of pinacol 1 to pinacolone $\mathbf{6}$ plus a water molecule, we note that the overall enthalpy of rearrangement $\Delta H_{p c}$ is negative and mostly on the small side, ranging from -0.82 to $-6.10 \mathrm{kcal} / \mathrm{mol}$. The relatively narrower range for the values for the $\Delta H_{p c}$ index is due to the net number of covalent bonds being preserved between reactant and products (not all, however, of the same type between reactant and products). The overall thermodynamic profile, include the transition state, is portrayed in figure 3 for three of the cases studied here $\left(\mathrm{R}=\mathrm{Me}, \mathrm{Pr}^{i}\right.$ and $\left.\mathrm{Ph}\right)$ giving

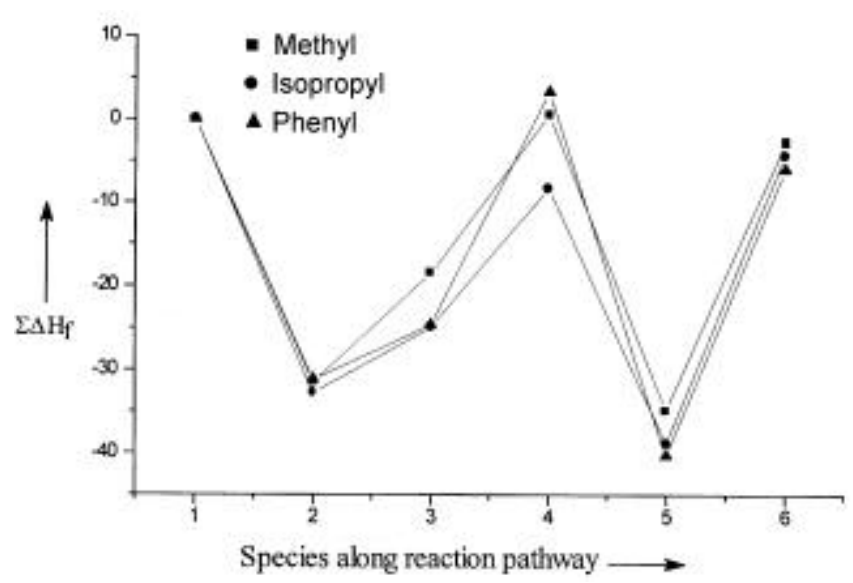

Figure 3. Overall thermodynamic profile for the pinacol-pinacolone rearrangement of 1,2,2-trimethyl-1,2-diols involving migration of methyl, isopropyl and phenyl groups. the six successive phases for the overall reaction in terms of the species $\mathbf{1}$ to $\mathbf{6}$ in turn, using the net sum of heats of formation $\Sigma \Delta H_{f}$ at each step along the way. We have scaled the profile such as to make the starting point for all three cases coincide with an arbitrary enthalpy value of zero. We note the overall trend is the same in all cases in that the peaks along the energy profile diagram include the starting pinacol 1, the transition state $\mathbf{4}$ and the pinacolone product $\mathbf{6}$ itself, while the lowest point is that of the rearranged cation $\mathbf{5}$.

\subsection{Relative migratory aptitudes}

Table 2 presents the $P M 3$ data concerning the actual rearrangement step itself, viz., the 1,2-migration of group $\mathrm{R}$ in species $\mathbf{3}$ to give the rearranged species $\mathbf{5}$ via the transition state $4\left(\mathrm{R}=\mathrm{H}, \mathrm{Me}, \mathrm{Et}, \mathrm{Pr}^{i}, \mathrm{Bu}^{t}, \mathrm{Ph}\right.$, 2-Py, 3-Py and 4-Py groups). The data includes the rearrangement enthalpy $\Delta H_{r}$, the activation energy $E_{a}$, the single negative Hessian eigenvalue $v_{i}$, and the net Mulliken charge $Q_{r}$ on the migrating group in the transition state.

As indicated earlier, the Wagner-Meerwein rearrangement of the carbocation $\mathbf{3}$ to carbocation $\mathbf{4}$ is here predicted to be an exothermic reaction for all nine cases. The negative values of the reaction enthalpy $\Delta H_{r}$ uniformly indicates a transition state on the "early" side, resembling the reactant rather than the product for all the cases, as per the Hammond postulate. This will be discussed in the section on transition state geometries.

Table 2. $P M 3$ data* for 1,2-shift of various migrating groups $\mathrm{R}$ in the Wagner-Meerwein step of the pinacolpinacolone rearrangement, giving reaction enthalpy $\Delta H_{r}$ for this step, the activation barrier $E_{a}$, the single Hessian negative eigenvalue $v_{i}$ and the net charge $Q_{r}$ on the migrating group $\mathrm{R}$ in the transition state.

\begin{tabular}{lcccc}
\hline $\mathrm{R}$ & $\Delta H_{r}$ & $E_{a}$ & $\mathrm{~V}_{I}$ & $Q_{r}$ \\
\hline $\mathrm{H}$ & $-15 \cdot 64$ & $14 \cdot 07$ & -1.152 & 0.0998 \\
$\mathrm{Me}$ & -16.22 & 19.12 & -0.658 & 0.1672 \\
$\mathrm{Et}$ & -14.92 & 18.91 & -0.274 & 0.2743 \\
$\mathrm{Pr}^{i}$ & -13.82 & $16 \cdot 81$ & -0.102 & 0.3062 \\
$\mathrm{Bu}^{t}$ & -13.28 & 14.66 & -0.178 & 0.3263 \\
$\mathrm{Ph}$ & -15.61 & 28.24 & -0.540 & 0.0963 \\
$2-\mathrm{Py}$ & -20.86 & 24.63 & -0.496 & 0.0663 \\
$3-\mathrm{Py}$ & -16.72 & 28.55 & -0.595 & 0.0593 \\
4-Py & -15.98 & 24.81 & -0.327 & 0.0627 \\
\hline
\end{tabular}

*Enthalpies in $\mathrm{kcal} / \mathrm{mol}$; Hessian eigenvalue in $\mathrm{mdyn} /$ ångstrom; charges in atomic units 
Thermodynamically, the rearrangement facility given by the enthalpy index $\Delta H_{r}$ turns out to be 2-Py $>3-\mathrm{Py}>\mathrm{Me}>4-\mathrm{Py}>\mathrm{H}>\mathrm{Ph}>\mathrm{Et}>\mathrm{Pr}^{i}>\mathrm{Bu}^{t}$. This order points to the negative effect that branching on the alkyl group has on thermodynamic facility, as well as to the relatively greater facility predicted for the three pyridyl groups. The case of hydride migration is somewhere in the middle. We also note that this ordering for thermodynamic facility does not correlate at all with the Mulliken charge index $Q_{r}$.

The enthalpy of activation $E_{a}$ for the migration step ranges from 14.07 to $28.55 \mathrm{kcal} / \mathrm{mol}$. Kinetically, the facility order for rearrangement with respect to the group $\mathrm{R}$ involved, as given by the activation energy $E_{a}$, is predicted to be $\mathrm{H}>\mathrm{Bu}^{t}>\operatorname{Pr}^{i}>\mathrm{Et}>$ $\mathrm{Me}>2-\mathrm{Py}>4-\mathrm{Py}>\mathrm{Ph}>3-\mathrm{Py}$. This predicted order of migration facility falls in good accordance with the limited experimental data ${ }^{15-17}$ available on the pinacol-pinacolone rearrangement, which gives the relative migratory aptitude in this situation $\mathrm{H}>$ $\mathrm{Me}>\mathrm{Ph}$. Other theoretical data ${ }^{18}$ on relative migratory aptitudes of some groups in a different cationic situation gives the ordering as $\mathrm{Bu}^{t}>\mathrm{Pr}^{i}>\mathrm{Et}>\mathrm{Me}$, much in line with these results of ours. This indicates that these gas phase calculations yield trends that fit well with those obtained experimentally in the solvent phase, justifying our use of the gas phase simulation here, at least insofar as qualititative trends are concerned.

Note that this ordering predicted for kinetic facility is quite different from that predicted for thermodynamic facility; the orderings are in fact often reverse to each other. The smallest group (hydride) is here predicted to migrate most easily, while the largest activation barrier is that for the 3-pyridyl group. The effect of branching in the alkyl groups here is to facilitate migration in the order $\mathrm{Bu}^{t}>$ $\mathrm{Pr}^{i}>\mathrm{Et}>\mathrm{Me}$. The aromatic groups $\mathrm{Ph}, 2-\mathrm{Py}, 3-\mathrm{Py}$ and 4-Py are predicted to have appreciably higher activation energies than those for the alkyl groups. We thus see that the aromatic groups rank lower than the alkyl groups in kinetic facility, but on the higher side with respect to thermodynamic facility.

The capacity of the migrating group to carry positive charge during migration has been suggested ${ }^{24}$ as a helpful factor for nucleophilic migrations within carbocation species, and is represented here by the Mulliken charge index $Q_{r}$. This index here has uniformly positive values, the order of magnitude with respect to group $\mathrm{R}$ being $\mathrm{Bu}^{t}>\mathrm{Pr}^{i}>\mathrm{Et}>\mathrm{Me}>\mathrm{H}>$ $\mathrm{Ph}>2$-Py $>4$-Py $>3$-Py. We observe here that the alkyl groups are predicted to have a greater capacity for carrying positive charge in the transition state than the aromatic groups. This capacity is indicated to correlate fairly well with the kinetic facility of migration (given by the ordering above derived from $E_{a}$ ), apart for the cases of hydride and phenyl group shifts. We may thus conclude that the capacity to bear positive charge in the transition state is one important factor that lowers the activation energy.

The factors discerned here which facilitate migration from the kinetic viewpoint are thus seen to be (a) whether the migrating group is aliphatic or aromatic, (b) degree of branching in the alkyl group, (c) capacity to carry positive charge, and (d) steric considerations as well. Steric bulk of the migrating group emerges as a major deciding factor for relative migratory aptitude of the phenyl, methyl and hydride groups, where the migration facility order $\mathrm{H}>\mathrm{Me}>\mathrm{Ph}$ holds.

\subsection{Transition state geometries}

Our PM3 values for determinants of geometry within the triangular moiety of the transition state $\mathbf{4}$ are given in table 3 , which includes the bond lengths, bond angles and dihedral as described earlier in figure 2, presented for the nine different $\mathrm{R}$ groups studied here. In general, the three carbon-carbon bonds marked as $R_{12}, R_{13}$ and $R_{23}$ (figure 2) have the following ranges: $R_{12}$ from 1.407 to $1.440 \AA, R_{13}$ from 1.949 to $2.158 \AA, \mathrm{R}_{23}$ from 1.931 to $2.471 \AA$, except for the hydride case, where the $R_{13}$ and $R_{23}$ bonds are carbon-hydrogen bonds ranging from 1.406 to $1.512 \AA$ in length, and where the bond lengths and bond angles indicate a roughly equilateral shape for the central triangular moiety. In all other cases, the $\mathrm{C} 1-\mathrm{C} 3$ and $\mathrm{C} 2-\mathrm{C} 3$ bond lengths are appreciably larger than single $\mathrm{C}-\mathrm{C}$ bond lengths, indicating the breaking and making of bonds. The length of the $\mathrm{C} 1-\mathrm{C} 2$ bond does not appreciably change in value between transition state, reactant or product, since it does not really participate in the reaction. The differences in the $\mathrm{C} 1-\mathrm{C} 3$ and $\mathrm{C} 2-\mathrm{C} 3$ bond lengths are not very large, and the triangular moiety is generally isosceles in shape, except for the $t$-butyl case, which more resembles a right-angled triangle. The case of the 2-Py group has the $\mathrm{C} 1-\mathrm{C} 3$ bond almost equal to the $\mathrm{C} 2-\mathrm{C} 3$ bond in length, while for the 4-Py case, the $\mathrm{C} 1-\mathrm{C} 3$ bond is shorter than the $\mathrm{C} 2-\mathrm{C} 3$ bond. Thus, for these cases, the transition state may seem 
Table 3. Geometry parameters* for the transition states of pinacol-pinacolone rearrangement involving various migrating groups (parameters described in text).

\begin{tabular}{|c|c|c|c|c|c|c|c|c|}
\hline $\mathrm{R}$ & $R_{12}$ & $R_{13}$ & $R_{23}$ & $\alpha$ & $\beta$ & $\gamma$ & $\varphi$ & $\varphi_{2}$ \\
\hline $\mathrm{H}$ & 1.441 & 1.406 & $1 \cdot 512$ & $64 \cdot 14$ & $56 \cdot 80$ & 59.04 & $86 \cdot 52$ & - \\
\hline $\mathrm{Me}$ & 1.433 & 1.949 & $2 \cdot 057$ & $73 \cdot 13$ & $65 \cdot 05$ & $41 \cdot 83$ & $89 \cdot 51$ & $-2 \cdot 37$ \\
\hline Et & 1.417 & 2.078 & $2 \cdot 144$ & $72 \cdot 89$ & 67.93 & $39 \cdot 18$ & $90 \cdot 69$ & -13.66 \\
\hline $\operatorname{Pr}^{i}$ & 1.407 & $2 \cdot 158$ & $2 \cdot 284$ & $76 \cdot 50$ & $66 \cdot 71$ & 36.79 & $86 \cdot 38$ & -14.06 \\
\hline $\mathrm{Bu}^{t}$ & 1.430 & $2 \cdot 005$ & $2 \cdot 471$ & $90 \cdot 40$ & $54 \cdot 24$ & $35 \cdot 36$ & $81 \cdot 03$ & $-42 \cdot 17$ \\
\hline $\mathrm{Ph}$ & 1.429 & 1.993 & 2.082 & 72.98 & $66 \cdot 13$ & 40.98 & $92 \cdot 05$ & 17.96 \\
\hline 2-Py & 1.433 & $2 \cdot 062$ & $2 \cdot 060$ & $69 \cdot 58$ & $69 \cdot 73$ & 40.69 & $96 \cdot 22$ & $18 \cdot 14$ \\
\hline 3-Py & 1.432 & $2 \cdot 004$ & $2 \cdot 046$ & $70 \cdot 88$ & $67 \cdot 74$ & $42 \cdot 38$ & $93 \cdot 35$ & $16 \cdot 36$ \\
\hline 4-Py & $1 \cdot 440$ & $2 \cdot 128$ & 1.931 & $62 \cdot 06$ & $76 \cdot 73$ & $41 \cdot 21$ & $101 \cdot 94$ & $10 \cdot 41$ \\
\hline
\end{tabular}

*All bond distances in ångstroms; bond angles and dihedral angles in degrees

relatively less "early" than the others, which is discussed later.

The dihedral $\varphi_{1}$ gives some idea of the threedimensional structure and configuration of the transition state by indicating how the 2-methyl group opposite the 1-hydroxy group relates to the planar triangular moiety. The values of this dihedral range from $81.03^{\circ}$ to $101.94^{\circ}$, thereby indicating that the plane incorporating the 2-methyl group is always approximately perpendicular to the triangular moiety described earlier.

The dihedral $\varphi_{2}$ describes the orientation of the migrating group $\mathrm{R}$ in relation to the triangular moiety $\mathrm{C} 1-\mathrm{C} 2-\mathrm{C} 3$. This angle is undefined for the hydride case, while for the methyl case it incorporates a methyl hydrogen atom, and a carbon atom for all the other cases. We note that the alkyl groups $\mathrm{Me}, \mathrm{Et}$, $\operatorname{Pr}^{i}$ and $\mathrm{Bu}^{t}$ give values of $\varphi_{2}$ varying from $-42 \cdot 17^{\circ}$ to $-2 \cdot 37^{\circ}$. In the context of the overall geometry (details not given in full here), this indicates that, for the methyl migration case, the methyl group has one hydrogen approximately in the plane of the triangular moiety, and the other two situated on opposite sides of the plane, much like a normal $s p^{3}$ hybridised methyl group might be expected to be. For the ethyl migration case, the ethyl group has its methyl group oriented away downwards from this plane, where $\varphi_{2}$ is $-13.66^{\circ}$, and its structure is largely that of a normal $s p^{3}$ hybridised ethyl group. The case of the migrating isopropyl group has its two methyl groups oriented approximately on opposites sides of this plane, and the overall conformation of the isopropyl group is much as would it be in a normal $s p^{3}$ situation. In analogous manner, the $t$ butyl group has one methyl group approximately along the plane of the triangular moiety, and the two others situated on opposite sides of this plane, and its overall structure accords with a normal $s p^{3}$ hybridised $\alpha$-carbon. We thus infer that the migrating alkyl group in all cases does not partake much of the planar nature of an alkyl carbocation having an $s p^{2}$ hybridised $\alpha$-carbon. The migrating group here rather bears more of the tetrahedral character of a usual alkyl group with an $s p^{3}$ hybridised $\alpha$-carbon. This points to a good degree of delocalisation of positive charge during the migration itself, which serves as a stabilising factor.

The aromatic rings of the migrating groups $\mathrm{Ph}, 2-$ Py, 3-Py and 4-Py are all orientated in almost the same way with respect to the triangular moiety. Their overall structure is more or less planar, indicating preservation of aromaticity. The dihedral $\varphi_{2}$ varies from $10.41^{\circ}$ to $18.14^{\circ}$ and indicates that these rings are nearly approximately coplanar with the triangular moiety $\mathrm{C} 1-\mathrm{C} 2-\mathrm{C} 3$. This co-planarity would certainly serve well to promote $\pi$-bond delocalisation between the aromatic rings and the triangular moiety (consisting of the breaking and forming bonds). Together with the above inference on the $s p^{3}$ character of the migrating alkyl groups, this recalls the theoretical study ${ }^{18}$ on the structure of $\pi$-complexes between various cations and an ethylene molecule, which predicts profuse orbital overlapping between the cation moiety and the ethylene $\pi$-bond leading to much delocalisation of positive charge and consequent stabilisation.

\subsection{Relative position of transition states}

Table 4 presents values of the ratio of the $\mathrm{C} 1-\mathrm{C} 3$ and $\mathrm{C} 2-\mathrm{C} 3$ bond lengths, viz. $R_{13} / R_{23}$, as it occurs in the transition states involved for the 1,2-migration of the nine different $\mathrm{R}$ groups studied. This ratio ranges from 0.8114 to 1.1020 in value, in most cases 
fairly close to unity, thus indicating that the transition states involved are neither too "early" or too "late", but somewhat in the middle insofar as this ratio itself would indicate. Relatively speaking though, most of the cases have the ratio value slightly less than unity, indicating a relatively "early" character for the corresponding transition states, in good line with what the Hammond postulate itself would indicate from the exothermicity of this reaction. The value of this ratio increases from the $t$-butyl case to that of the 4-pyridyl group in the order: $\mathrm{Bu}^{t}<\mathrm{H}<\mathrm{Pr}^{i}<\mathrm{Me}<\mathrm{Ph}<\mathrm{Et}<3-\mathrm{Py}<2-\mathrm{Py}<$ 4-Py, which may be taken as indicating the relative degree of "earliness" of the transition state in that order insofar as the bond length ratio index is concerned.

When comparing the values of this ratio index to the values of the enthalpy index $\Delta H_{r}$, we note that, while this enthalpy index is uniformly negative, indicating an "early" transition state for all cases, the bond length ratio index itself is not uniformly smaller than unity. This indicates that the bond length ratio index is useful only to make relative assignments of position along the reaction path, and not as an absolute index for such. There is, however, some degree of correspondence between the bond ratio index and the enthalpy index, notably for the cases of the $\mathrm{Bu}^{t}, \mathrm{Pr}^{i}, \mathrm{Me}, \mathrm{Ph}, \mathrm{Et}, 3-\mathrm{Py}$ and 2-Py groups, where the relative ordering predicted for degree of "earliness" is the same from both indices, viz. $\mathrm{Bu}^{t}>\mathrm{Pr}^{i}>\mathrm{Me}>\mathrm{Ph}>\mathrm{Et}<3-\mathrm{Py}>2-\mathrm{Py}$.

The case for the $t$-butyl group has the "earliest" transition state from both the geometrical and ther-

Table 4. The ratio of lengths of the bonds breaking and making in the transition states involved in the 1,2-migration of group $\mathrm{R}$ for the various systems studied along with remarks on the relative position of the transition state along the reaction pathway.

\begin{tabular}{lccccc}
\hline $\mathrm{R}$ & $R_{13}$ & $R_{23}$ & $R_{13} / R_{23}$ & $H_{r}$ & Remarks \\
\hline $\mathrm{H}$ & 1.406 & 1.512 & 0.9299 & -15.64 & Quite early \\
$\mathrm{Me}$ & 1.949 & 2.057 & 0.9474 & -16.22 & - \\
$\mathrm{Et}$ & 2.078 & 2.144 & 0.9692 & -14.92 & - \\
$\mathrm{Pr}^{i}$ & 2.158 & 2.284 & 0.9448 & -13.82 & - \\
$\mathrm{Bu}^{t}$ & 2.005 & 2.471 & 0.8114 & -13.28 & Most early \\
$\mathrm{Ph}$ & 1.993 & 2.082 & 0.9572 & -15.61 & $\begin{array}{c}\text { Earlier than } \\
\text { pyridyls }\end{array}$ \\
& & & & & \\
2-Py & 2.062 & 2.060 & 1.0009 & -20.86 & \\
3-Py & 2.004 & 2.046 & 0.9794 & -16.72 & - \\
4-Py & 2.128 & 1.931 & 1.1020 & -15.98 & Most late \\
\hline
\end{tabular}

*All bond distances in ångstroms modynamic considerations, with a value of 0.8114 for the bond length ratio index, as well as the least degree of exothermicity $\left(\Delta H_{r}=-13.28 \mathrm{kcal} / \mathrm{mol}\right)$. The case for the 2-Py group may be designated as relatively "late" from both considerations, where the bond length ratio index is 1.0009 (closest to a "midway" position insofar as the bond ratio index is concerned) and the enthalpy index is $-20.86 \mathrm{kcal} /$ mol (most exothermic).

The bond length ratio index would serve well as an absolute indicator of position along the reaction coordinate only if the substitution of the 1- and 2carbons was symmetrical, viz., if there were two 1-methyl groups instead of one 1-methyl and one 1hydroxy group. The presence of the 1-hydroxy group upsets this symmetry, because of which the bond length ratio index can serve only as an indicator of relative position along the reaction path, and not of absolute position.

\section{Conclusions}

This theoretical study leads to the following predictions and conclusions:

(1) The Wagner-Meerwein 1,2-migration in the pinacol-pinacolone rearrangement is invariably favoured thermodynamically, regardless of the migrating group involved.

(2) The relative migratory aptitude from the kinetic viewpoint is influenced by the basic type of the group (whether aliphatic or aromatic), degree of branching in the alkyl group, capacity of the group to carry positive charge, and steric considerations.

(3) The order predicted here for relative migratory aptitude is $\mathrm{H}>\mathrm{Bu}^{t}>\mathrm{Pr}^{i}>\mathrm{Et}>\mathrm{Me}>2-\mathrm{Py}>4-\mathrm{Py}>$ $\mathrm{Ph}>3$-Py which well reflects the limited experimental data available for these systems as well as theoretical data on similar systems.

(4) The migrating alkyl groups have basically $s p^{3}$ hybridised $\alpha$-carbons, while the migrating aryl groups are coplanar with the rearranging moiety in the transition state. These observations infer that there would be much delocalisation of positive charge in the transition states leading to net stabilisation.

(5) The application of geometrical and thermodynamic considerations lead to the following consistent order, viz. $t$-butyl $>$ isopropyl $>$ methyl $>$ phenyl $>$ ethyl > 2-pyridyl > 4-pyridyl, for relative "earliness" of the transition states involved, in good accordance with the Hammond postulate. 


\section{Acknowledgement}

One of the authors (ZP) is grateful to the Directorate of Higher and Technical Education, Government of Mizoram, for a Research Scholarship.

\section{References}

1. Olah G A and Schleyer P v R 1969 Carbonium ions (New York, London, Sydney, Toronto: Wiley-Interscience) vol. 2; Olah G A and Schleyer P v R 1971 Carbonium ions (New York, London, Sydney, Toronto: Wiley-Interscience) vol. 3; Creary X 1991 Chem. Rev. 911625

2. Vogel P 1985 Organic chemistry: Studies in organic chemistry 21 (New York: Elsevier Science) ch. 10; Saunders M and Jiminez-Vazquez H A 1991 Chem. Rev. 91375

3. Pine S H 1987 Organic chemistry 5th edn (Singapore: McGraw-Hill) p. 973

4. Sykes P 1986 A guide book to mechanism in organic chemistry 6th edn (New Delhi: Orient Longman) p. 111

5. Fittig W 1859 Annalen 110 23; Fittig W 1860 Annalen 11454

6. Collins C J 1960 Q. Rev. 375

7. Cecchi P, Cipollini R, Pizzabiocca A, Renzi G and Speranza M 1988 Tetrahedron 444847
8. Suzuki K, Ohkuma T, Miyazawa M and Tsichihashi G 1986 Tetrahedron Lett. 27373

9. Suzuki K, Katayama E and Tsuchihashi G 1984 Tetrahedron Lett. 251817

10. Suzuki K, Katayamma E and Tsuchihashi G 1983 Tetrahedron Lett. 244997

11. Stiles M and Meyer R P 1959 J. Am. Chem. Soc. 81 1499

12. Shono $T$, Fujita $K$, Kumai $S$, Watanabe $T$ and Nishiguchi I 1972 Tetrahedron Lett. 313249

13. Pilkington J W and Waring A J 1973 Tetrahedron Lett. 444345

14. Tsuchihashi G, Tomooka K and Suzuki K 1984 Tetrahedron Lett. 254253

15. Shine H J 1967 Aromatic rearrangements (Amsterdam, London, New York: Elsevier) ch. 1

16. Collins C J 1955 J. Am. Chem. Soc. 775517

17. Carlin R B and Shivaramakrishnan K P 1970 J. Org. Chem. 352368

18. Mayo P D 1980 Rearrangement in ground and excited states (New York: Academic Press) p. 35

19. Hammond G S 1955 J. Am. Chem. Soc. 77334

20. Stewart J J P 1989 J. Comput. Chem. 10209

21. Stewart J J P 1983 QCPE Bull. 343

22. Shano D F J 1985 Opt. Theory Appl. 46 87; Stewart J J P 1990 Frank J Seiler Research Laboratory, United States Air Force Academy, CO 80840

23. Maquestiau A, Flammang R, Flammang-Barbieux M and Mispreuve H 1980 Tetrahedron 361994

24. Young-Sook P H 1988 Bull. Korean Chem. Soc. 10151 